\title{
Short-Term Wind Speed Hybrid Forecasting Model Based on Bias Correcting Study and Its Application
}

\author{
Mingfei Niu, Shaolong Sun, Jie Wu, and Yuanlei Zhang \\ School of Mathematics and Statistics, Lanzhou University, Lanzhou 730000, China \\ Correspondence should be addressed to Shaolong Sun; sunshl13@lzu.edu.cn
}

Received 27 October 2014; Revised 7 December 2014; Accepted 8 December 2014

Academic Editor: Pandian Vasant

Copyright ( 2015 Mingfei Niu et al. This is an open access article distributed under the Creative Commons Attribution License, which permits unrestricted use, distribution, and reproduction in any medium, provided the original work is properly cited.

\begin{abstract}
The accuracy of wind speed forecasting is becoming increasingly important to improve and optimize renewable wind power generation. In particular, reliable short-term wind speed forecasting can enable model predictive control of wind turbines and realtime optimization of wind farm operation. However, due to the strong stochastic nature and dynamic uncertainty of wind speed, the forecasting of wind speed data using different patterns is difficult. This paper proposes a novel combination bias correcting forecasting method, which includes the combination forecasting method and forecasting bias correcting model. The forecasting result shows that the combination bias correcting forecasting method can more accurately forecast the trend of wind speed and has a good robustness.
\end{abstract}

\section{Introduction}

Because of the global energy shortage, renewable energy has received increasing attention, just now. Wind power is one of the cleanest renewable energy sources that produces no greenhouse gases, has no effect on climate change, and produces little environmental impacts, and the energy generated from the wind has been well recognized as environmentally friendly, socially beneficial, and economically competitive for many applications [1]. As of now the effectiveness of wind speed forecasting is an important role in the scheduling of wind power. At present, these methods can be divided into two categories: statistical models and machine-learning models. Statistical models primarily use a time series approach and have been successfully applied for forecasting [2-7]. These models are based on the assumption that a linear correlation structure exists among time series values. Therefore, nonlinear patterns cannot be captured using these models. To overcome this limitation, machinelearning models have been used to improve nonlinear time series predictions (which primarily include artificial neural networks, support vector machines, heuristic algorithm, and fuzzy logic methods) [8-34].

In a nutshell, in the past decades, many computational intelligence techniques have been developed for short-term wind speed forecasting, for instance, support vector regression $[15,27,35]$, support vector machine $[26,31,33,36]$, fuzzy model $[21,27]$, artificial neural networks $[8,9,11,13$, 14, $20,23-25,28,29]$, wavelet method $[9,34,37]$, and heuristic intelligence algorithm: particle swarm optimization [37, 38], adaptive particle swarm optimization [17, 36, 39], chaotic particle swarm optimization [31], biogeography-based optimization [10], coral reefs optimization [16], gravitational search algorithm [19], and harmony search algorithm [16]. In the next section, we will detail the explanation of the previous work in the short-term wind speed prediction.

The remaining sections are arranged as follows. The related work will be brief description in Section 2. The preparation methods and main modeling process are described from Section 3 to Section 6. Section 7 forecasts the wind speed of Penglai using three wind farms and provides the forecasting results and analyses. Finally, the conclusion is presented in Section 8 and the future research in Section 9.

\section{Related Work}

In the above references, Song et al. [5] employ a discrete-state Markov chain to model the nonlinear characteristic of the wind speed time series, and a Bayesian inference is applied 
to evaluate the parameters of the Markov-switching model. Finally, by comparison with other methods, this proposed method outperforms them. Liu et al. [9] present four important decomposing algorithms including wavelet decomposition, wavelet packet decomposition, empirical mode decomposition, and fast ensemble empirical mode decomposition, which are all adopted to realize the wind speed high-precision predictions. Salcedo-Sanz et al. [16] introduce a new hybrid coral reefs optimization and harmony search algorithm; this novel approach is utilized to obtain the best set of meteorological variables in the context of short-term wind speed forecasting, and the selection variable will be input to an extreme learning machine network. Experimental result shows that these proposed methods have good results when compared to other approaches. Wang et al. [17] proposed an optimization model to decide the rated power system and the capacity of a compressed air energy storage system in a power system with high wind power penetration. Moreno et al. [18] proposed a strategy including the uncertainty of involving market and wind power. Mondal et al. [19] solved economic dispatch problem in wind generation. In a nutshell, as the randomness of wind speed distribution, every prediction model owns some limitations.

In the short-term wind speed forecasting, because of ignoring of the secondary influence factors and correlations, every prediction model can generate prediction errors, which are the difference between the predicted value and the actual value, the main causes that forecasting method just considers the main factors, and many of the secondary factors are ignored. However, as the effect of the secondary factors, the forecasting bias may form a certain trend. Making allowance for these minor influence factors, the bias correction becomes important. The basic idea of forecasting bias correction is following. After forecasting by the prediction model and comparing with the actual wind speed, forecasting error is generated. Using suitable prediction model to forecast error, error correction can be got, which is used to modify the original forecasting result. The error correction prediction model expression is as follows: $Y=Y_{c}+\hat{e}_{c} \cdot Y$ is final forecasting value, $Y_{c}$ is combination forecasting value, and $\widehat{e}_{c}$ is bias correction.

Nowadays, there are some error correction models [36, 40-42], such as the periodic extrapolation, vector error correction model, partial simulation approximate value, and Bayesian error correction model. But the relevant researches about the short-term wind speed and wind power error correction models are very rare. So this paper quotes bias correction model in short-term wind speed forecasting, thus, making wind power scheduling reasonable.

Due to the randomness of wind speed, the former forecasting models have their own limitations. It is because of the volatility of the wind speed; firstly, this paper proposes a combination of a linear model and two nonlinear models: double exponential smoothing method (DES), backpropagation of particle swarm optimization artificial neural network (PSO-BPANN), and Elman artificial neural network (Elman$A N N$ ). The inputs of PSO-BPANN and Elman-ANN consist of historical wind speed data and residual errors of the $D E S$ model. Then the combined weight will use adaptive particle swarm optimization algorithm (APSO) to optimize. The combination model can more accurately forecast shorttime wind speed. The reasons of wind speed forecasting errors are analyzed; then, the empirical orthogonal function model will be error correction. Making use of this model, main variables can be extracted, and error correction model is built by the empirical orthogonal function regression method. Some advantages are that the main variables are determined by the properties of wind speed series itself, but not prior artificial regulation, and can reflect the actual wind speed data basic structure, and expansion equation converges fast. Finally, combination bias correcting forecasting model is presented. In order to check the validity of the model, the case study will be analyzed in detail.

Generally speaking, in this research, our main contribution is that we set up the combination bias correction forecasting model in the short-term wind speed forecasting, which consists of double exponential smoothing (DES), PSO$B P$ artificial neural network, and Elman artificial neural network and, finally, adaptive particle swarm optimization algorithm (APSO) to optimize the combination weights. Forecasting error can be corrected by the empirical orthogonal function, which can be used in variables analysis and regression forecasting for wind speed prediction bias and correcting wind speed prediction result. In this paper, the tenminute wind speed data from three wind farms in Penglai of Shandong province in China were used as examples to evaluate the performance of the proposed approach. To avoid volatility due to the PSO-BP, Elman, and PSO optimization algorithm, all of the simulations were repeated 50 times prior to averaging. As time goes on, more wind speed information will be obtained, more accurate wind speed characteristic will be derived by forecasting models, and the new information on the wind speed is absorbed by this combination bias correction model. Therefore, the performance of this combination bias correction model will be accurate and stable.

\section{Combination Forecasting Model}

Due to the random features of wind speed, the nonlinear characteristics are significant. So the combination forecasting consists of a linear model and two nonlinear models; then the combined weight will use adaptive particle swarm optimization algorithm (APSO) to optimize. Three models theories are as follows.

3.1. Wind Speed DES Model. Exponential smoothing technique used to forecast wind speed is comparatively simple. It only needs a single wind speed series. It can be divided into single exponential smoothing method (SES), double exponential smoothing method (DES), cubic exponential smoothing method (CES), and so on. The main steps of exponential smoothing method used to forecast wind speed include modeling and calculating the consequence of exponential smoothing, determining the smoothing coefficient of $\alpha$, and forecasting. Because DES is based on single exponential smoothing values and is more accurate than the single exponential smoothing method, it can better reflect the 
linear characteristic of wind speed. So this paper uses DES. The procedure is as follows.

3.1.1. Modeling and Calculating the Consequence of Exponential Smoothing. The DES is modeled by the wind speed data, and the expression is as follows:

$$
\widehat{x}_{l+T}=\widehat{a}_{T}+\widehat{b}_{T} l .
$$

The DES model is similar to the double moving average method (DMA); first of all, on the base of the sequence of the single exponential smoothing (smoothing coefficient $0<\alpha<$ 1),

$$
S_{t}^{(1)}=\alpha x_{t}+(1-\alpha) S_{t-1}^{(1)}, \quad t=1,2, \ldots, T,
$$

and calculating the consequence of second exponential smoothing

$$
S_{t}^{(2)}=\alpha S_{t}^{(1)}+(1-\alpha) S_{t-1}^{(2)}, \quad t=1,2, \ldots, T,
$$

where the initial value $S_{0}{ }^{(2)}=S_{0}{ }^{(1)}$ and $S_{0}{ }^{(1)}=x_{1}, S_{1}^{(1)}=$ $\alpha x_{1}+(1-\alpha) S_{0}{ }^{(1)}=x_{1}, S_{0}{ }^{(2)}=S_{1}{ }^{(1)}=x_{1}$.

\subsubsection{Determining the Smoothing Coefficient $\alpha$ and Forecast-} ing. A sequence of the single exponential smoothing $S_{t}^{(1)}$ is relative to data $x_{t}$ migration or lag effect, and a sequence of the double exponential smoothing $S_{t}{ }^{(2)}$ is relative to $S_{t}{ }^{(1)}$ lag effect, otherwise. Under certain conditions, such as $t$ large enough, especially $\alpha$ is close to 1 , and two lags are equal. Among them, the smoothing coefficient $\alpha$ can be optimized via analyzing the wind speed forecasting error. Just for the sake of smooth sequence, $\alpha$ can be small enough. If it is used to predict, when the original wind speed volatility is not obvious, $\alpha$ can get smaller or get bigger, in order to make the smoothing sequence reflect the changes of the wind speed data.

Through calculation we get the intercept and slope of the prediction linear

$$
\begin{gathered}
\widehat{a}=2 S_{t}^{(1)}-S_{t}^{(2)} \\
\widehat{b}=\frac{\alpha}{1-\alpha}\left[S_{t}^{(1)}-S_{t}^{(2)}\right], \quad t=1,2, \ldots, T .
\end{gathered}
$$

Finally, prediction model is as follows:

$$
\begin{gathered}
\bar{x}_{t+1}=\widehat{a}_{t}+\widehat{b}_{t}=\frac{2-\alpha}{1-\alpha} S_{t}^{(1)}-\frac{1}{1-\alpha} S_{t}^{(2)}, \quad t=1,2, \ldots, T-1 \\
\widehat{x}_{T+l}=\widehat{a}_{T}+\widehat{b}_{T} l, \quad l=1,2, \ldots
\end{gathered}
$$

3.2. Wind Speed PSO-BP Neural Network Prediction Model. In order to solve the nonlinear features of wind speed, artificial neural network ( $A N N)$ methods have been proposed. $A N N$ is able to give better performance in dealing with the nonlinear relationships among their input variables [35]. The conventional backpropagation algorithm $(B P)$ is successfully applied to complex nonlinear problems. However, using BP method needs the following; the transfer function of each neuron must be different. Moreover, it has been proven that gradient techniques are slow to train and are sensitive to the initial guess which can possibly be trapped in a local minimum [43].

To overcome these shortcomings, the paper introduces particle swarm optimization algorithm (PSO) to optimize the $B P$ network to solve the wind speed forecasting problem. The PSO algorithm is applied to the neural network in the training phase, to obtain a set of weights that will minimize the error function in competitive time. Weights are progressively updated until the convergence criterion is satisfied. The objective function to be minimized by the PSO algorithm is the predicted error function [37].

Figure 1 shows the flow process of PSO-BPANN forecasting model [38].

In Figure 1, the normalized formula and fitness function are as follows:

$$
y_{i}=\frac{x_{i}-x_{\min }}{x_{\max }-x_{\min }}
$$

where $x_{i}$ is defined as an initial group of data among the collected wind speed data groups. $x_{\max }$ and $x_{\min }$ express the maximum and minimum data group among the collected data groups, respectively; consider

$$
f=\frac{1}{n} \sum_{j=1}^{n} \sum_{k=1}^{m}\left(y_{k}-t_{k}\right)^{2} \text {, }
$$

where $y_{k}$ is the actual forecasting output value, $t_{k}$ is the target value, $n$ is the numbers of training sample, $m$ is the nodes of output.

In short-term wind speed forecasting, the sample data is normalized and smoothly processed. Firstly, the inputs of PSO-BPANN are made of historical wind speed and residual errors calculated by DES model. The target output is original value of wind speed next ten-minute data.

\subsection{Wind Speed Elman Artificial Neural Network Prediction} Model. This paper has proposed another neural network: Elman artificial neural network. The Elman neural network is proposed firstly by Elman in 1990 [44, 45]. It is a form of recurrent neural network $(R N N)$ by adding recurrent links into hidden layer as a feedback connection which allows the network to learn to recognize and generate temporal patterns. However, $R N N$ has some merits for nonlinear system modeling and forecasting while the order of systems under consideration is unknown or with uncertainty. Generally, comparing with the $B P$ neural networks, the training for the Elman recursion neural networks is faster than for the $B P$ neural networks.

The researches on Elman-ANN have been developed with nonlinear modeling, transfer function, and field of application [46, 47], such as nonlinear stable adaptive control and solar activity forecasting.

The flow chart of Elman-ANN prediction model is shown in Figure 2 [48]. 


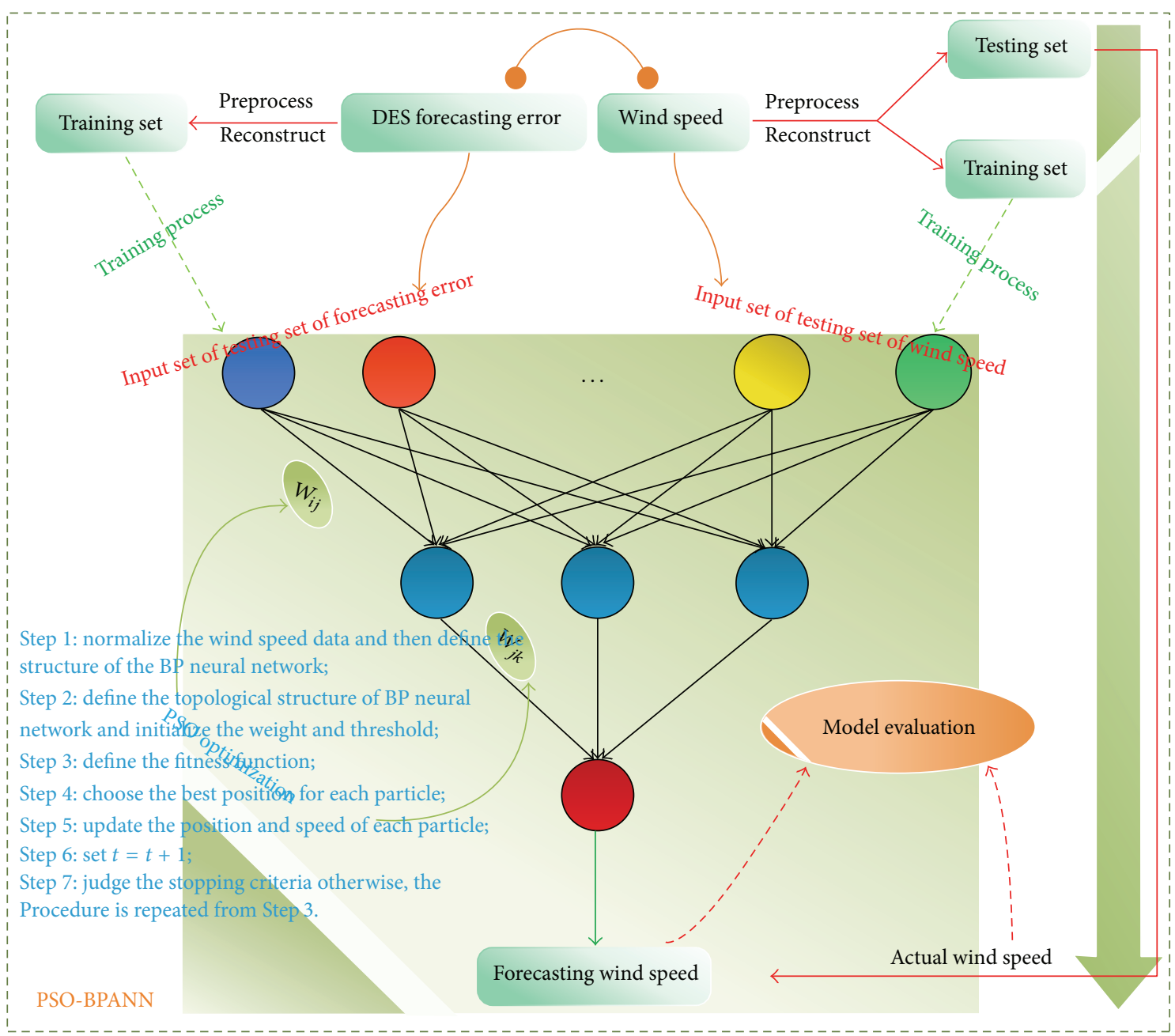

FIGURE 1: The process of PSO-BPANN model.

In Figure 2 the correction formula is as follows:

$$
\omega_{i j}{ }^{(l+1)}=\omega_{i j}{ }^{(l)}-\eta \frac{\partial E_{k}}{\partial \omega_{i j}{ }^{(l)}}, \quad \eta>0,
$$

wherein $\omega_{i j}{ }^{(l)}$ is the weight coefficient between $j$ neuron in $l$ layer and $i$ neuron in $l+1$ layer; $\eta$ is gain.

In this paper, we have processed the wind speed data sample according to neighboring three ten-minute time periods and residual errors calculated by DES model before the training of the network, aiming to forecast one-step ahead wind speed by using previous three ten-minute data and residual errors.

\section{Combination Forecasting Model}

DES prediction is $y_{1}$; PSO-BPANN prediction is $y_{2}$; ElmanANN prediction is $y_{3}$; weighted average prediction is $y_{c}$. Combination forecasting model is as follows:

$$
y_{c}=\omega_{1} y_{1}+\omega_{2} y_{2}+\omega_{3} y_{3}
$$

wherein $\omega_{1}, \omega_{2}$, and $\omega_{3}$ are weights, respectively. And the following is satisfied:

$$
\omega_{1}+\omega_{2}+\omega_{3}=1
$$

In order to improve the precision of the combination forecasting model, the paper proposed a novel adaptive particle swarm optimization (APSO) to optimize the combination weights. As the change of particle fitness function value, inertia weight will be automatically adjusted, which makes the particle search direction illuminating enhancement. The APSO algorithm not only converges fast, but also does not fall into local extreme points easily. The specific formula of adaptive adjustment is as follows [49]:

$$
\begin{gathered}
v_{i}(t+1)=\omega(t) v_{i}(t)+c_{1} r_{1}\left[p_{i}(t)-x_{i}(t)\right] \\
+c_{2} r_{2}\left[p_{g}(t)-x_{i}(t)\right], \\
x_{i}(t+1)=x_{i}(t)+v_{i}(t+1),
\end{gathered}
$$




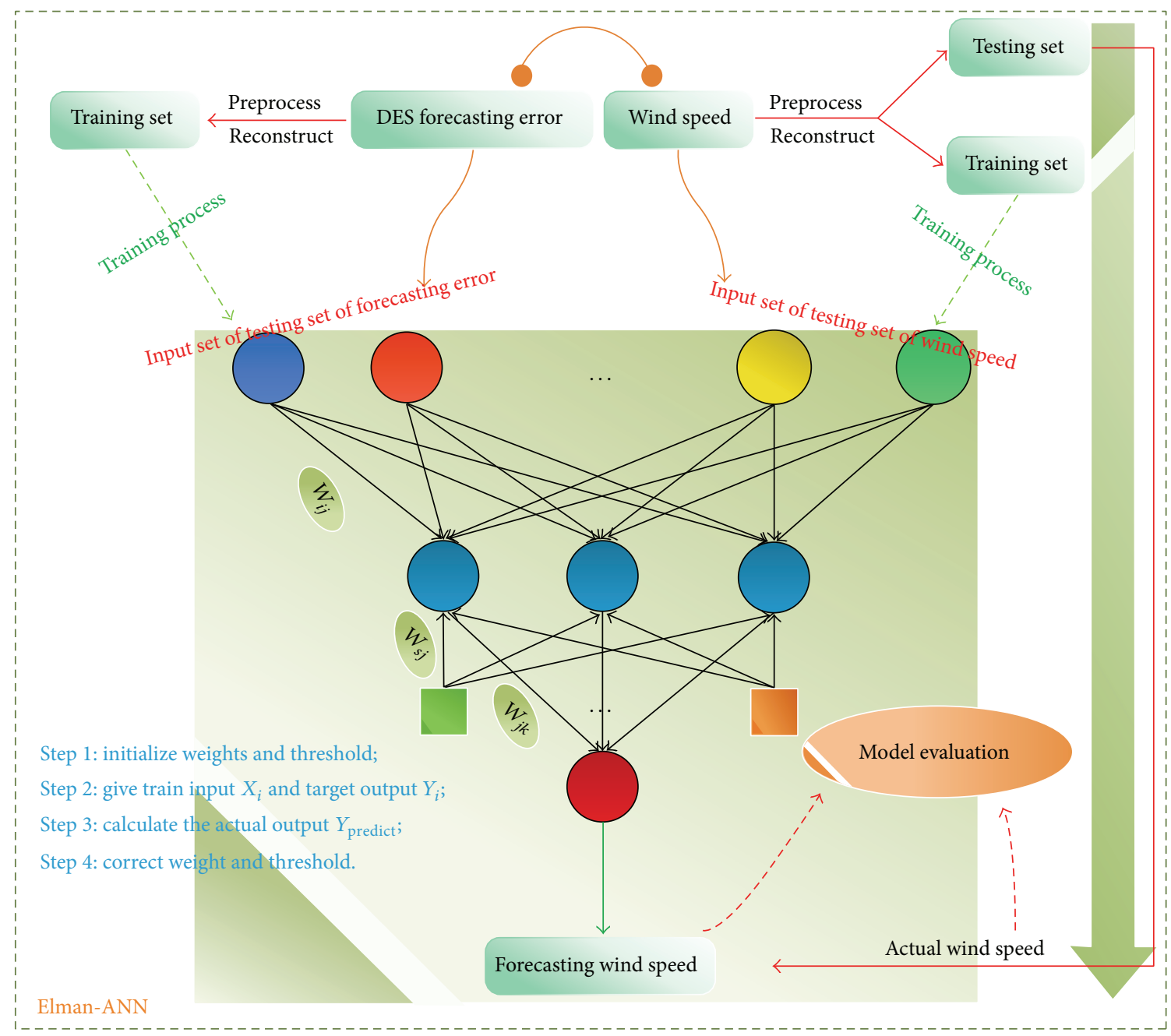

FIGURE 2: The process of Elman-ANN model.

$\omega(t)$

$$
= \begin{cases}\lambda \cdot \omega(t-1) & \\ +\theta \frac{f\left(p_{g}(t)\right)-f\left(x_{i}(t)\right)}{f\left(p_{g}(t)\right)-f\left(x_{\min }(t)\right)} & \text { if } \omega(t) \geq \omega_{\min } \\ \omega_{\min } & \text { otherwise, }\end{cases}
$$

where $c_{1}$ and $c_{2}$ are positive constants, which are called acceleration coefficients. $r_{1}$ and $r_{2}$ are two random numbers in the range $[0,1] . x_{i}=\left(x_{i}(1), x_{i}(2), \ldots, x_{i}(D)\right)$ represents the $i$ th particle. $p_{i}=\left(p_{i}(1), p_{i}(2), \ldots, p_{i}(D)\right)$ represents the best previous position (the position giving the best fitness value) of the $i$ th particle. The symbol $g$ represents the index of the best particle among all the particles in the population. $v_{i}=\left(v_{i}(1), v_{i}(2), \ldots, v_{i}(D)\right)$ represents the rate of the position change for particle $i$ and $D$ represent the search space dimension. $\omega(t)$ is the adaptive inertia weight, $\lambda$ and $\theta$ are constraint factors in the range $[0,1], \omega_{\min }$ is the minimum inertia weight, and $f(\bullet)$ is the fitness function. In this paper, the fitness function is defined as follows:

$$
f=-\frac{1}{N} \sum_{i=1}^{N}\left(y_{c}-\widehat{y}_{i}\right)^{2},
$$

where $y_{c}$ and $\widehat{y}_{i}$ are the output value of combination model and the actual wind speed value; $N$ is the sample size.

The process of APSO to optimize the combination weights is in Figure 3 [39].

\section{Prediction Error Correction Model-EOF Model}

Empirical orthogonal function $(E O F)$ has recently become popular tool in the atmospheric science decomposition and expansion since its introduction several decades ago [50, 51]. It is equivalent to the principal component used in the multivariate statistics and is close relatives to the bases used in factor analysis. It is characterized by the fast convergence 

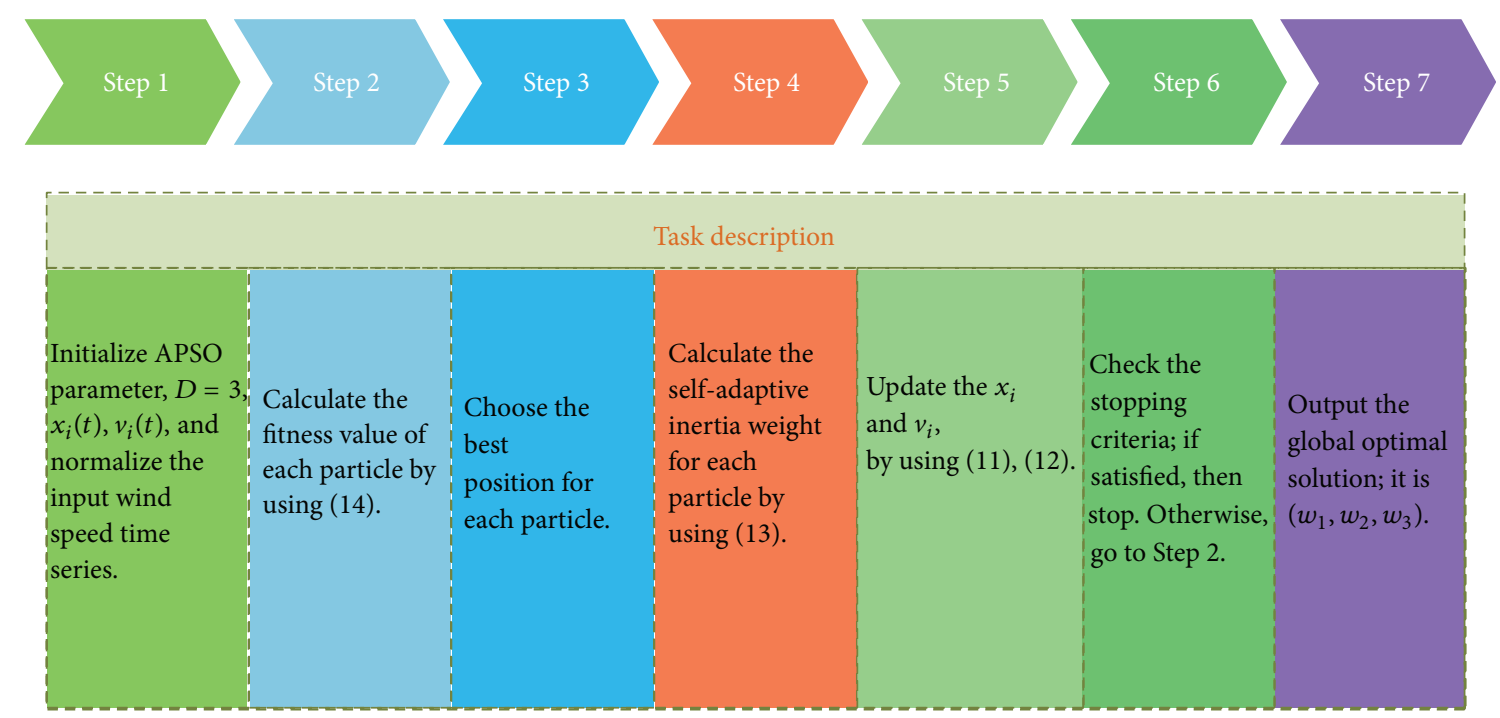

FIGURE 3: The process of APSO optimization combination weights.

of expansion and can approach variable field condition by a few items. It was first proposed by Pearson in 1902 [52], and Lorenz first introduced it to the weather and climate research [53]. At present, EOF has been widely used in the ocean and other fields. Probably the most important thing is that this method often enables a description of the variations of a complex geophysical field with a relatively small number of functions and associated time coefficients. This property is especially important in the development of statistical prediction schemes which rely on multiple linear regression, since the skill and statistical confidence of those schemes depend heavily upon a priori methods of reducing the number of available predictors $[54,55]$.

In this paper, we will describe and illustrate the EOF technique, and the forecasting errors have been corrected with $E O F$ decomposition and regression.

5.1. EOF Decomposition. EOF is also called empirical orthogonal decomposition or natural orthogonal decomposition. It is similar to principal component analysis but different from meanings and analysis methods [48]. The basic idea is to decompose data that contain $m$ variables in $n$ time observation field. That is to say, the wind speed forecasting error $e_{i j}(i=1,2, \ldots, m, j=1,2, \ldots, n$ that contain $m$ variables in $n$ time observation field of a region) is decomposed into the product of orthogonally the sum of space function $s_{i p}$ and the sum of function $t_{p j}$. The form is as follows [56]:

$$
\begin{array}{r}
e_{i j}=\sum_{p=1}^{m} s_{i p} t_{p j}=s_{i 1} t_{1 j}+s_{i 2} t_{2 j}+\cdots+s_{i m} t_{m j}, \\
(i=1,2, \ldots, m, j=1,2, \ldots, n) .
\end{array}
$$

It is written in the matrix form:

$$
\mathbf{E}=\mathbf{S T} .
$$

According to the orthogonality, the decomposition should be satisfied:

$$
\begin{gathered}
s_{i} s_{j}=\sum_{p=1}^{m} s_{i p} s_{p j}= \begin{cases}0 & i \neq j \\
1 & i=j\end{cases} \\
t_{i} t_{j}=\sum_{p=1}^{m} t_{i p} t_{p j}= \begin{cases}0 & i \neq j \\
1 & i=j .\end{cases}
\end{gathered}
$$

Formula (15) is right multiplied $\mathbf{E}^{T}$ :

$$
\mathbf{E E}^{T}=\mathbf{S T T}^{T} \mathbf{S}^{T} .
$$

Denote

$$
\mathbf{A}=\mathbf{E E}^{T}
$$

A is a $m \times m$ symmetric matrix, According to the principle of symmetric matrix decomposition, the following must be satisfied:

$$
\mathbf{S}^{T} \mathbf{A S}=\boldsymbol{\Lambda} \quad \text { or } \quad \mathbf{A}=\mathbf{S} \boldsymbol{\Lambda} \mathbf{S}^{T},
$$

wherein $\boldsymbol{\Lambda}$ is a diagonal matrix made up of $\mathbf{A}$ eigenvalues. $\mathbf{S}$ is a matrix made up of the feature vector. So

$$
\begin{gathered}
\mathbf{T T}^{T}=\boldsymbol{\Lambda} \\
\mathbf{S}^{T} \mathbf{S}=\mathbf{S S}^{T}=\mathbf{I},
\end{gathered}
$$

where $\mathbf{I}$ is a unit matrix. $\mathbf{S}$ can be got from $\mathbf{A}$. The time function can be got by (15) left multiplied $\mathbf{S}^{T}$ :

$$
\mathbf{T}=\mathbf{S}^{T} \mathbf{E} \quad \text { or } \quad t_{i j}=\sum_{p=1}^{m} s_{p i} e_{p j} .
$$

From (15)-(21) completed the EOF decomposition as like the equation (15). 


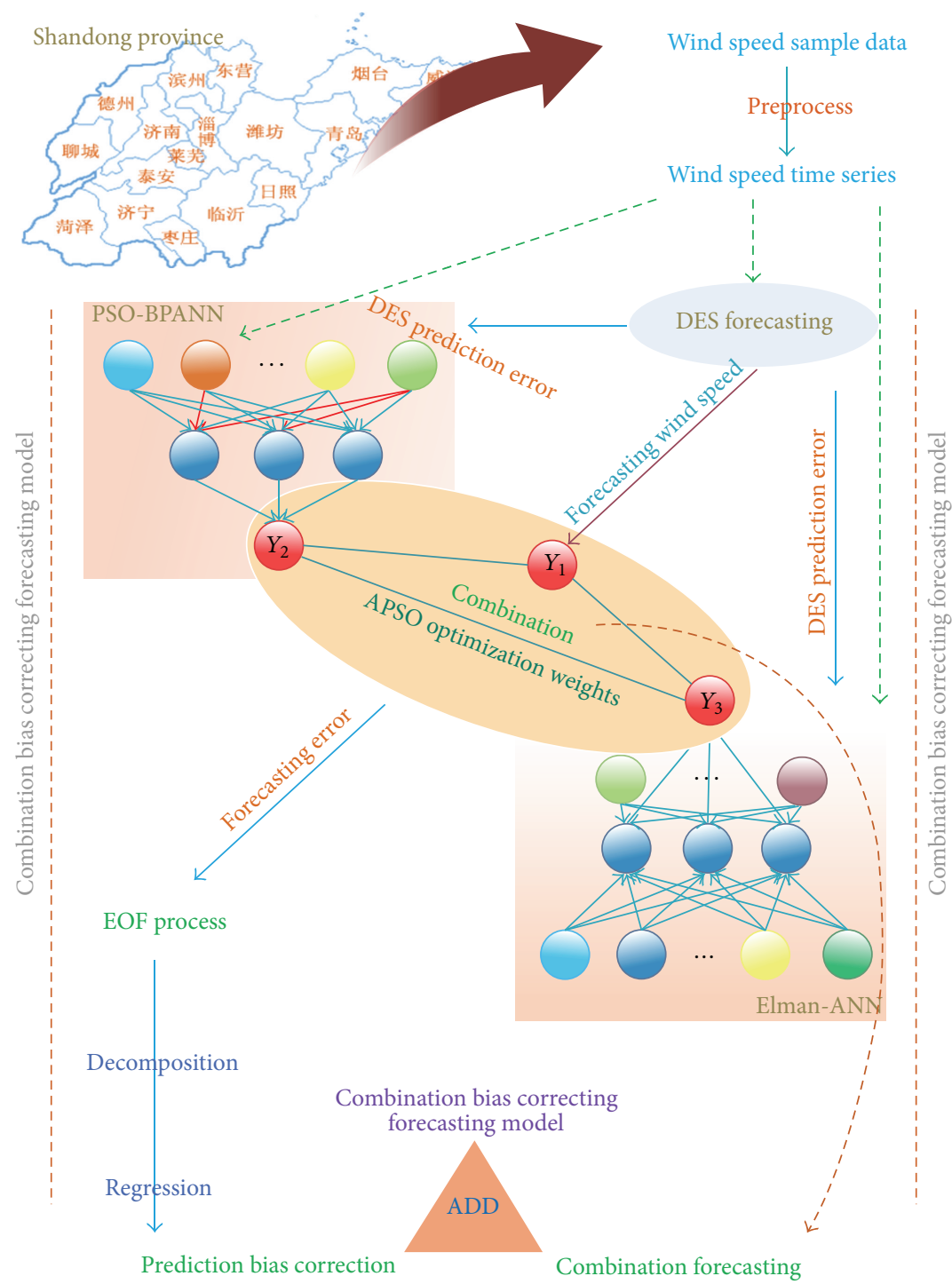

FIGURE 4: The process of combination bias correcting forecasting model.

5.2. EOF Regression. The main idea of EOF regression is that, decomposed into orthogonal principal component, EOF model completes regression with a small amount of principal components and changes into orthogonal variables. There is no large error on regression equation [48].

With the EOF decomposition of $\mathbf{E}$, according to the precision requirement, the former $p$ variance components can be extracted $X_{1}, X_{2}, \ldots, X_{p}$. The regression equation is as follows:

$$
e_{i j}=\beta_{0}+\beta_{1} X_{1}+\cdots+\beta_{p} X_{p}
$$

In the regression equation (23), the selection of $p$ is very important. The characteristics of $\lambda_{i}(i=1,2, \ldots, n, n$ is the numbers of eigenvalue) are calculated by the wind speed forecasting error covariance matrix $\mathbf{E E}^{T}$; let the former $p$ eigenvalue be in descending order, and calculation formula is as follows:

$$
\varepsilon^{2}=\frac{\sum_{i=1}^{p} \lambda_{i}}{\sum_{i=1}^{m} \lambda_{i}}
$$

According to the precision requirement, $p$ can be selected, usually $\varepsilon^{2} \geq 80 \%$.

\section{Combination Bias Correcting Forecasting Model}

Combination bias correcting forecasting model consists of the combination forecasting model and the forecasting error correcting model. Figure 4 shows the flow chart of modeling. 
TABLE 1: Models evaluation of simulation.

\begin{tabular}{lcccccc}
\hline \multirow{2}{*}{ Wind farm } & \multirow{2}{*}{ Evaluation criteria } & \multicolumn{4}{c}{ Simulation } \\
& & DES & PSO-BPNN & Elman & Hybrid & Hybrid bias correcting \\
\hline \multirow{2}{*}{1} & MAPE (\%) & 6.45 & 3.07 & 2.96 & 2.01 & 1.26 \\
& MSE & 0.511 & 0.056 & 0.040 & 0.056 & 0.019 \\
\hline \multirow{2}{*}{5} & MAPE (\%) & 7.12 & 3.14 & 2.30 & 2.05 & 0.97 \\
& MSE & 0.575 & 0.062 & 0.059 & 0.051 & 0.027 \\
\hline
\end{tabular}

\section{Case Study}

7.1. Collection of Data. The data of three farms in Penglai of China are collected to examine the combination bias correcting model. In particular, firstly, the data of wind farm 1 are used to witness the whole process of the proposed method. In the same way, the corresponding forecasting results of wind farm 2 and wind farm 5 are shown and further verify the validity of the method, respectively.

In this paper, the 4500 observations of ten-minute wind speed are used for training, and the remaining 6 observations are used to test the effectiveness of the models. It is used to forecast next one hour; the wind speed times series sampled each hour consisting of 6 data points.

7.2. Evaluation Criteria of Forecast Performance. In order to evaluate the forecast effectiveness of the model, two indices for error forecast serve as the criteria to evaluate the forecasting performance. They are mean square error (MSE) and mean absolute percent error (MAPE). The values of the indices are smaller and the forecast performance is better. The indices are as follows [57]:

$$
\begin{aligned}
\text { MSE } & =\frac{1}{n} \sum_{t=1}^{n}\left|Y_{t}-\widehat{Y}_{t}\right| \\
\text { MAPE } & =\frac{1}{n} \sum_{t=1}^{n} \frac{\left|Y_{t}-\widehat{Y}_{t}\right|}{Y_{i}},
\end{aligned}
$$

wherein $Y_{t}$ is the value of actual wind speed for a time period $t$ and $\widehat{Y}_{t}$ is the forecast value for the same period.

7.3. Combination Bias Correcting Forecasting Model. To verify the proposed combination bias correction method, firstly, we conducted a simulation experiment with the different wind farm. In this experiment, we compared our model with the DES, PSO-BPNN, Elman, and combined model, which were established using the original data without error correction. The comparisons of wind speed values simulate using the $D E S$, the PSO-BPNN, Elman, and the combined model with the actual models being shown in Figure 5. Specific process is as follows.

DES prediction is $y_{1}$; PSO-BPANN prediction is $y_{2}$; Elman-ANN prediction is $y_{3}$; weighted average prediction is $y_{c}$. Prediction errors are $e_{1}, e_{2}, e_{3}$, and $e_{c}$. Combination forecasting model is $y_{c}=\omega_{1} y_{1}+\omega_{2} y_{2}+\omega_{3} y_{3}$, where APSO optimization weights are $\omega_{1}, \omega_{2}$, and $\omega_{3}$, and $\omega_{1}+\omega_{2}+\omega_{3}=1$.
Finally, combination bias correcting forecasting is $\widehat{y}_{c}$, EOF decomposition and regression are $\widehat{e}_{c}$, and $\widehat{y}_{c}=y_{c}+\widehat{e}_{c}$.

To avoid randomness due to the PSO-BP, Elman, and PSO optimization algorithm, all of the simulations were repeated 50 times prior to averaging. For more instinctive to compare, Figure 5 shows the DES model, PSO-BPANN model, Elman$A N N$, combination forecasting model, and combination bias correcting simulation forecasting graphics at the same time. By the error graphics it can be seen that the combination bias correcting forecasting method is better than combination forecasting method, and combination method is better than single forecasting method.

In order to accurately describe the simulation effect of this proposed model. From Table 1, it can be seen that the combination bias correcting model performs much better than combination forecasting model and single forecasting model. More precisely, in wind farm 1, comparing with DES, PSO-BPANN, Elman-ANN, and combination model, MAPE and MSE of the proposed model are reduced to $1.26 \%$ and 0.019 . However, in order to reflect the robustness of this model, wind farm 2 and wind farm 5 are also analyzed; comparing with DES, PSO-BPANN, Elman-ANN, and combination model, MAPE and MSE of the proposed model are reduced to $0.97 \%$ and $0.027,1.41 \%$ and 0.021 , respectively. Therefore, using this model to forecast is very feasible.

7.4. The Analysis of Forecasting Results. Based on the historical data of three wind farms' simulation training, the shortterm wind speed forecasting is reasonable. To avoid volatility due to the PSO-BP, Elman, and PSO optimization algorithm, all of the forecasting was repeated 50 times prior to averaging.

Figure 6 shows the short-term wind speed forecasting graphics of three wind farms and the covariance matrix table of the EOF decomposition. In the covariance matrix table, $P_{1}, P_{2}, P_{3}$, and $P_{4}$ are components. The variance contribution rate is defined $\varphi=\lambda_{i} / \sum_{j=1}^{m} \lambda_{j}$, and the cumulative variance contribution rate is defined $\varepsilon^{2}=\sum_{i=1}^{p} \lambda_{i} / \sum_{i=1}^{m} \lambda_{i}$, and the number of eigenvalues is $m$.

According to the precision requirement, $p$ can be selected, usually $\varepsilon^{2} \geq 80 \%$; in wind farm 1 , the rate of $\lambda_{1}$ and $\lambda_{2}$ is $99.22 \%$, and $P_{3}$ is ignored; then $p=2$. With this similarity, the rate of $\lambda_{1}$ is $81.57 \%$ and $93.57 \%$, respectively; then $p=1$ of wind farm 2 and wind farm 5 .

Form Table 2 and Figure 6 it is clear that the combination bias correcting model performs much better than 


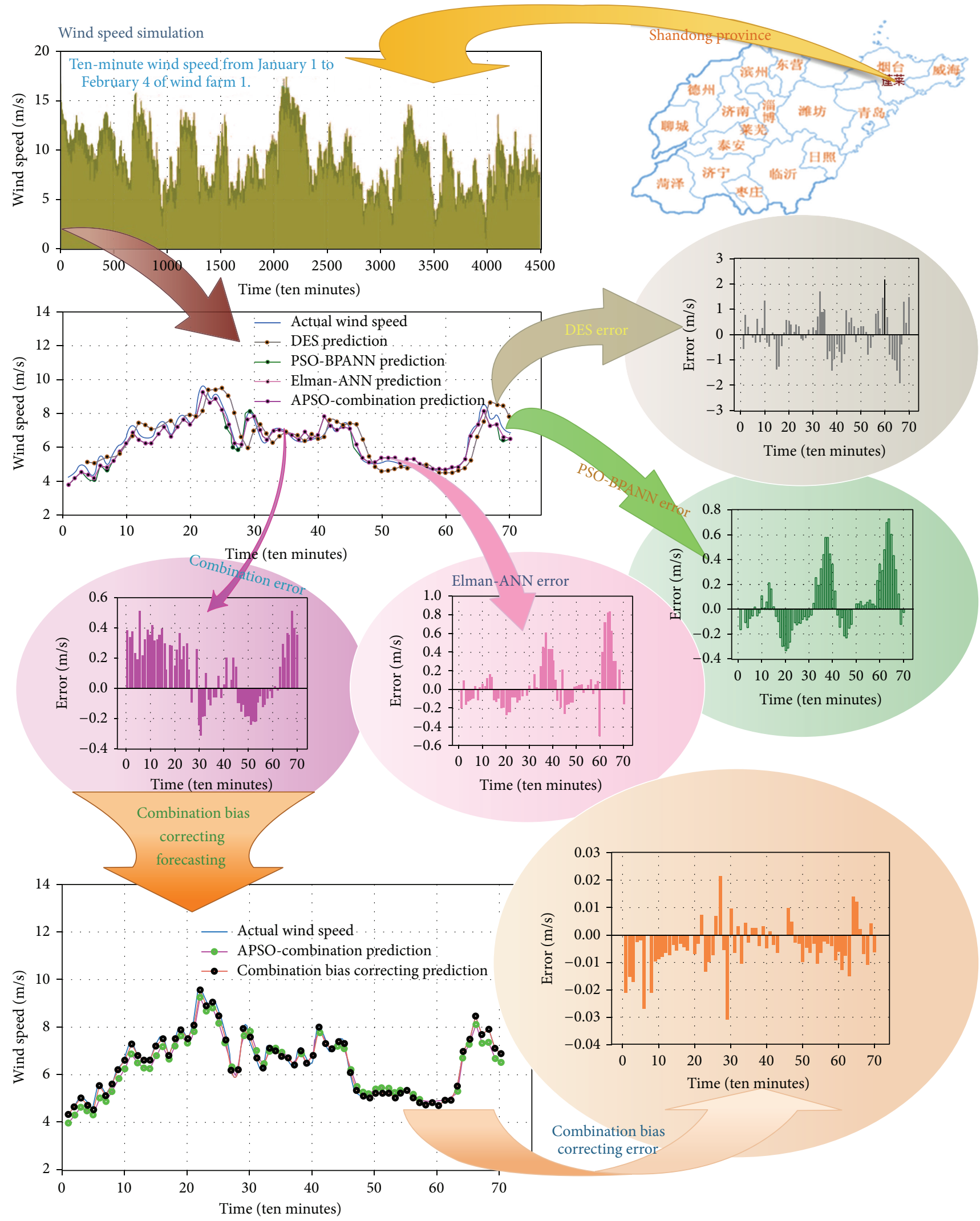

FIGURE 5: Simulation chart of wind farm 1. 

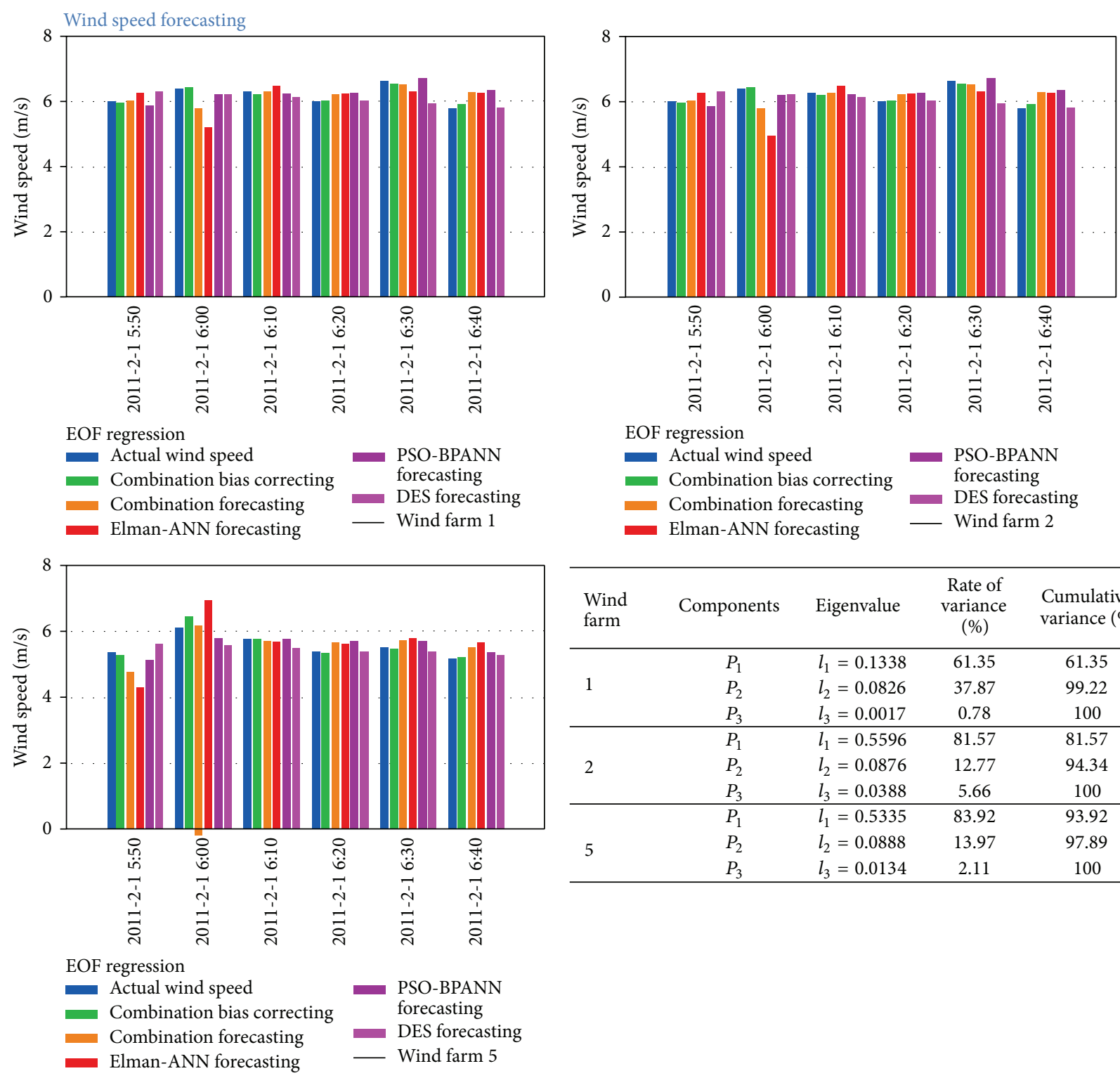

\begin{tabular}{lcccc}
\hline $\begin{array}{l}\text { Wind } \\
\text { farm }\end{array}$ & Components & Eigenvalue & $\begin{array}{c}\text { Rate of } \\
\text { variance } \\
(\%)\end{array}$ & $\begin{array}{c}\text { Cumulative } \\
\text { variance (\%) }\end{array}$ \\
\hline \multirow{3}{*}{1} & $P_{1}$ & $l_{1}=0.1338$ & 61.35 & 61.35 \\
& $P_{2}$ & $l_{2}=0.0826$ & 37.87 & 99.22 \\
& $P_{3}$ & $l_{3}=0.0017$ & 0.78 & 100 \\
\hline \multirow{2}{*}{2} & $P_{1}$ & $l_{1}=0.5596$ & 81.57 & 81.57 \\
& $P_{2}$ & $l_{2}=0.0876$ & 12.77 & 94.34 \\
& $P_{3}$ & $l_{3}=0.0388$ & 5.66 & 100 \\
\hline \multirow{3}{*}{5} & $P_{1}$ & $l_{1}=0.5335$ & 83.92 & 93.92 \\
& $P_{2}$ & $l_{2}=0.0888$ & 13.97 & 97.89 \\
& $P_{3}$ & $l_{3}=0.0134$ & 2.11 & 100 \\
\hline
\end{tabular}

FIGURE 6: Short-term wind speed forecasting.

TABLE 2: Models evaluation of forecasting.

\begin{tabular}{|c|c|c|c|c|c|c|}
\hline \multirow{2}{*}{ Wind farm } & \multirow{2}{*}{ Evaluation criteria } & \multicolumn{5}{|c|}{ Forecasting } \\
\hline & & DES & PSO-BPNN & Elman & Hybrid & Hybrid bias correcting \\
\hline \multirow{2}{*}{1} & MAPE (\%) & 6.61 & 3.72 & 3.08 & 2.75 & 1.27 \\
\hline & MSE & 0.111 & 0.050 & 0.056 & 0.043 & 0.137 \\
\hline \multirow{2}{*}{2} & MAPE (\%) & 3.89 & 3.58 & 7.67 & 3.84 & 2.12 \\
\hline & MSE & 0.107 & 0.072 & 0.429 & 0.112 & 0.043 \\
\hline \multirow{2}{*}{5} & MAPE (\%) & 3.98 & 4.52 & 9.35 & 5.81 & 1.19 \\
\hline & MSE & 0.079 & 0.065 & 0.402 & 0.139 & 0.035 \\
\hline
\end{tabular}

combination forecasting model and single forecasting model. More precisely, in wind farm 1, comparing with DES, PSO$B P A N N$, Elman-ANN, and combination model, MAPE and $M S E$ of the proposed model are reduced to $1.27 \%$ and 0.137 . By comparing the MAPE and MSE of the combination bias correction forecasting model they are also the smallest. But it can be shown that this combination bias correction model can be just right for wind farm 1 . In order to eliminate the randomness, this paper also forecast wind farm 2 and wind farm 5 . 
Similarly, in order to reflect the steadiness of this combination bias correction forecasting model, we analyze the forecasting results of wind farm 2 and wind farm 5 in detail. For wind farm 2, comparing with DES, PSO-BPANN, Elman$A N N$, the combination model, and the proposed model, MAPE is $3.89 \%, 3.58 \%, 7.67 \%, 3.84 \%$, and $2.12 \%$, respectively, and MSE is $0.107,0.072,0.429,0.112$, and 0.043 , respectively. In a similar way, comparing with DES, PSO-BPANN, Elman$A N N$, the combination model and this proposed model for wind farm 5, MAPE is 3.98\%, $4.52 \%, 9.35 \%, 5.81 \%$, and $1.19 \%$, respectively, and MSE is $0.079,0.065,0.402,0.139$, and 0.035 , respectively.

Through the above analysis, in detail, we used this test to evaluate the predictive performances of the five models. In the three wind farms, it is shown that the combination bias correcting forecasting method is better than combination forecasting method, and combination method is better than single forecasting method; the MAPE and MSE of the optimized hybrid bias correction model are also the smallest. Hence, all of the indices imply that the optimized hybrid bias correction model can effectively decrease the error of the forecasting values compared to the other four forecasting methods.

In a word, by the detailed analysis of three wind farms, the combination bias correcting forecasting method can more accurately forecast the trend of wind speed; $E O F$ decomposition and regression are an effective bias correction tool that may be combined with other forecasting methods, such as statistical and other artificial intelligence models, and have a good performance, which can be applied to the nonstationary wind speed forecasting.

\section{Conclusion}

Due to the importance of the wind speed forecasting error, this paper proposes a novel combination bias correcting forecasting method, which includes the combination forecasting method and forecasting bias correcting model. The method can improve the precision of forecasting. The main conclusions are as follows. (1) The combination forecasting model consists of DES, PSO-BP artificial neural network model, and Elman artificial neural network model for shortterm wind speed forecasting which are proposed and APSO to optimize the combination weights. Forecasting results are more satisfactory than the single forecasting model. (2) EOF forecasting bias correction method can be used in variables analysis and regression forecasting for wind speed prediction bias and correcting wind speed prediction result. (3) By the detailed analysis of the forecasting results of three wind farms, the combination bias correcting forecasting method can more accurately forecast the trend of wind speed and has a good robustness.

\section{Future Research}

In the short-term wind speed prediction, because of the wind speed nonlinear and chaotic characteristics we will be preprocessing the original data. Using wavelet transform, empirical mode decomposition, or singular spectrum analysis, removes white noise of the original wind speed series, and the filtered series will be modeling. However, the parameters of this preprocessing method can be optimized by the novel heuristic intelligence algorithm; for example, the parameters (windows length $L$ and component $r$ ) of the singular spectrum analysis are optimized by the gravitational search algorithm, firefly algorithm or shuffled leapfrog algorithm, and so on. In addition, this hybrid method also can be applied in other fields, such as power load forecasting, stock index forecasting, air transport demand forecasting, accidental deaths series forecasting, and exchange rates forecasting in the future.

\section{Conflict of Interests}

The authors declare that there is no conflict of interests regarding the publication of this paper.

\section{Acknowledgments}

This research was supported by the National Natural Science Foundation of China (Grant no. 71171102). The authors are grateful to the editors and reviewers for their valuable suggestions.

\section{References}

[1] M. Monfared, H. Rastegar, and H. M. Kojabadi, "A new strategy for wind speed forecasting using artificial intelligent methods," Renewable Energy, vol. 34, no. 3, pp. 845-848, 2009.

[2] G. D’Amico, F. Petroni, and F. Prattico, "Wind speed and energy forecasting at different time scales: a nonparametric approach," Physica A, vol. 406, pp. 59-66, 2014.

[3] H. Liu, H.-Q. Tian, and Y.-F. Li, "Comparison of two new ARIMA-ANN and ARIMA-Kalman hybrid methods for wind speed prediction," Applied Energy, vol. 98, pp. 415-424, 2012.

[4] A. Tascikaraoglu and M. Uzunoglu, "A review of combined approaches for prediction of short-term wind speed and power," Renewable and Sustainable Energy Reviews, vol. 34, pp. 243-254, 2014.

[5] Z. Song, Y. Jiang, and Z. Zhang, "Short-term wind speed forecasting with Markov-switching model," Applied Energy, vol. 130, pp. 103-112, 2014.

[6] M. Zhou, Z. Yan, Y. X. Ni et al., "A novel ARIMA approach on electricity price forecasting with the improvement of predicted error," Proceeding of the CSEE, vol. 24, no. 12, pp. 63-68, 2004.

[7] J. Aghaei, T. Niknam, R. Azizipanah-Abarghooee, and J. M. Arroyo, "Scenario-based dynamic economic emission dispatch considering load and wind power uncertainties," International Journal of Electrical Power and Energy Systems, vol. 47, no. 1, pp. 351-367, 2013.

[8] Y. Jiang, Z. Song, and A. Kusiak, "Very short-term wind speed forecasting with Bayesian structural break model," Renewable Energy, vol. 50, pp. 637-647, 2013.

[9] H. Liu, H.-Q. Tian, and Y.-F. Li, "Comparison of new hybrid FEEMD-MLP, FEEMD-ANFIS, Wavelet Packet-MLP and Wavelet Packet-ANFIS for wind speed predictions," Energy Conversion and Management, vol. 89, pp. 1-11, 2015.

[10] R. A. Gupta, R. Kumar, and A. K. Bansal, “BBO-based small autonomous hybrid power system optimization incorporating 
wind speed and solar radiation forecasting," Renewable and Sustainable Energy Reviews, vol. 41, pp. 1366-1375, 2015.

[11] J.-Z. Wang, J.-J. Wang, Z.-G. Zhang, and S.-P. Guo, "Forecasting stock indices with back propagation neural network," Expert Systems with Applications, vol. 38, no. 11, pp. 14346-14355, 2011.

[12] H. Liu, H.-Q. Tian, C. Chen, and Y.-F. Li, "A hybrid statistical method to predict wind speed and wind power," Renewable Energy, vol. 35, no. 8, pp. 1857-1861, 2010.

[13] J. Hu, J. Wang, and G. Zeng, "A hybrid forecasting approach applied to wind speed time series," Renewable Energy, vol. 60, pp. 185-194, 2013.

[14] J.-J. Wang, J.-Z. Wang, Z.-G. Zhang, and S.-P. Guo, "Stock index forecasting based on a hybrid model," Omega, vol. 40, no. 6, pp. 758-766, 2012.

[15] J. Wang, W. Zhu, W. Zhang, and D. Sun, "A trend fixed on firstly and seasonal adjustment model combined with the $\varepsilon$-SVR for short-term forecasting of electricity demand," Energy Policy, vol. 37, no. 11, pp. 4901-4909, 2009.

[16] S. Salcedo-Sanz, A. Pastor-Sánchez, J. Del Ser, L. Prieto, and Z. W. Geem, "A coral reefs optimization algorithm with harmony search operatorsfor accurate wind speed prediction," Renewable Energy, vol. 75, pp. 93-101, 2015.

[17] J. Wang, S. Zhu, W. Zhang, and H. Lu, "Combined modeling for electric load forecasting with adaptive particle swarm optimization," Energy, vol. 35, no. 4, pp. 1671-1678, 2010.

[18] M. Á. Moreno, M. Bueno, and J. Usaola, "Evaluating riskconstrained bidding strategies in adjustment spot markets for wind power producers," International Journal of Electrical Power and Energy Systems, vol. 43, no. 1, pp. 703-711, 2012.

[19] S. Mondal, A. Bhattacharya, and S. H. Nee Dey, "Multi-objective economic emission load dispatch solution using gravitational search algorithm and considering wind power penetration," International Journal of Electrical Power and Energy Systems, vol. 44, no. 1, pp. 282-292, 2013.

[20] K. W. Chau, "Particle swarm optimization training algorithm for ANNs in stage prediction of Shing Mun River," Journal of Hydrology, vol. 329, no. 3-4, pp. 363-367, 2006.

[21] C. T. Cheng, C. P. Ou, and K. W. Chau, "Combining a fuzzy optimal model with a genetic algorithm to solve multi-objective rainfall-runoff model calibration," Journal of Hydrology, vol. 268, no. 1-4, pp. 72-86, 2002.

[22] P. Flores, A. Tapia, and G. Tapia, "Application of a control algorithm for wind speed prediction and active power generation," Renewable Energy, vol. 30, no. 4, pp. 523-536, 2005.

[23] Z. X. Guo, W. K. Wong, and M. Li, "Sparsely connected neural network-based time series forecasting," Information Sciences, vol. 193, pp. 54-71, 2012.

[24] C. Hamzaçebi, "Improving artificial neural networks' performance in seasonal time series forecasting," Information Sciences, vol. 178, no. 23, pp. 4550-4559, 2008.

[25] S. A. Kalogirou, "Artificial neural networks in renewable energy systems applications: a review," Renewable and Sustainable Energy Reviews, vol. 5, no. 4, pp. 373-401, 2000.

[26] J.-Y. Lin, C.-T. Cheng, and K.-W. Chau, "Using support vector machines for long-term discharge prediction," Hydrological Sciences Journal, vol. 51, no. 4, pp. 599-612, 2006.

[27] K.-P. Lin, P.-F. Pai, Y.-M. Lu, and P.-T. Chang, "Revenue forecasting using a least-squares support vector regression model in a fuzzy environment," Information Sciences, vol. 220, pp. 196-209, 2013.
[28] M. Carolin Mabel and E. Fernandez, "Analysis of wind power generation and prediction using ANN: a case study," Renewable Energy, vol. 33, no. 5, pp. 986-992, 2008.

[29] N. Muttil and K.-W. Chau, "Neural network and genetic programming for modelling coastal algal blooms," International Journal of Environment and Pollution, vol. 28, no. 3-4, pp. 223238, 2006.

[30] A. Sfetsos, "A comparison of various forecasting techniques applied to mean hourly wind speed time series," Renewable Energy, vol. 21, no. 1, pp. 23-35, 2000.

[31] Q. Wu, R. Law, E. Wu, and J. X. Lin, "A hybrid-forecasting model reducing Gaussian noise based on the Gaussian support vector regression machine and chaotic particle swarm optimization," Information Sciences, vol. 238, pp. 96-110, 2013.

[32] J.-X. Xie, C.-T. Cheng, K.-W. Chau, and Y.-Z. Pei, "A hybrid adaptive time-delay neural network model for multi-stepahead prediction of sunspot activity," International Journal of Environment and Pollution, vol. 28, no. 3-4, pp. 364-381, 2006.

[33] J. Y. Zhou, J. Shi, and G. Li, "Fine tuning support vector machines for short-term wind speed forecasting," Energy Conversion and Management, vol. 52, no. 4, pp. 1990-1998, 2011.

[34] L.-J. Wang, L. Dong, X.-Z. Liao, and Y. Gao, "Short-term power prediction of a wind farm based on wavelet analysis," Proceedings of the Chinese Society of Electrical Engineering, vol. 29, no. 28, pp. 30-33, 2009.

[35] K. Chen and J. Yu, "Short-term wind speed prediction using an unscented Kalman filter based state-space support vector regression approach," Applied Energy, vol. 113, pp. 690-705, 2014.

[36] Y.-S. Huang, J.-J. Deng, and Z.-Z. Yuan, "SVM short-term load forecasting based on ARMA error calibration and the adaptive particle swarm optimization," Power System Protection and Control, vol. 39, no. 14, pp. 26-32, 2011.

[37] Z. A. Bashir and M. E. El-Hawary, "Applying wavelets to shortterm load forecasting using PSO-based neural networks," IEEE Transactions on Power Systems, vol. 24, no. 1, pp. 20-27, 2009.

[38] D. Xian and X. Bingji, "Telecommunication traffic forecasting based on BP neural network trained by PSO," Journal of Central South University, vol. 42, no. 1, pp. 24-25, 2011.

[39] C. Dong, G. Wang, Z. Chen, and Z. Yu, "A method of selfadaptive inertia weight for PSO," in Proceedings of the International Conference on Computer Science and Software Engineering (CSSE '08), vol. 1, pp. 1195-1198, IEEE, Hubei, China, December 2008.

[40] C. Xiu, T. Wang, M. Tian, Y. Li, and Y. Cheng, "Shortterm prediction method of wind speed series basedon fractal interpolation," Chaos, Solitons \& Fractals, vol. 68, pp. 89-97, 2014.

[41] K. G. Sheela and S. N. Deepa, "Neural network based hybrid computing model for wind speed prediction," Neurocomputing, vol. 122, pp. 425-429, 2013.

[42] M. Xu, Y. Qiao, and Z. Lu, "A comprehensive error evaluation method for short-term wind power prediction," Automation of Electric Power Systems, vol. 35, no. 12, pp. 20-26, 2011.

[43] D. E. Rumelhart, G. E. Hinton, and R. J. Williams, "Learning representations by back-propagating errors," Nature, vol. 323, no. 6088, pp. 533-536, 1986.

[44] J. L. Elman, "Finding structure in time," Cognitive Science, vol. 14, no. 2, pp. 179-211, 1990.

[45] H.-T. He and X. Tian, "An improved Elman network and its application in flatness prediction modeling," in Proceedings 
of the 2nd International Conference on Innovative Computing, Information and Control (ICICIC '07), vol. 9, p. 552, September 2007.

[46] S. Marra and F. C. Morabito, "A new technique for solar activity forecasting using recurrent Elman networks," World Academy of Science, Engineering and Technology, vol. 7, no. 8, pp. 68-73, 2005.

[47] X. Li, Z. Chen, and Z. Yuan, "Nonlinear stable adaptive control based upon Elman networks," Applied Mathematics-A Journal of Chinese Universities, vol. 15, no. 3, pp. 332-340, 2000.

[48] X. Nan, Q. Li, D. Qiu, Y. Zhao, and X. Guo, "Short-term wind speed syntheses correcting forecasting model and its application," International Journal of Electrical Power and Energy Systems, vol. 49, no. 1, pp. 264-268, 2013.

[49] J. Wang, S. Zhu, W. Zhang, and H. Lu, "Combined modeling for electric load forecasting with adaptive particle swarm optimization," Energy, vol. 35, no. 4, pp. 1671-1678, 2010.

[50] A. M. Obukhov, "Statistically homogeneous fields on a sphere," Uspekhi Matematicheskikh Nauk, vol. 2, pp. 196-198, 1947.

[51] J. E. Kutzbach, "Empirical eigenvectors of sea-level pressure, surface temperature and precipitation complexes over North America," Journal of Applied Meteorology, vol. 6, no. 5, pp. 791802, 1967.

[52] K. Pearson, "On lines and plans of closest fit to system of points in space philosophical," Magazine, vol. 6, pp. 559-572, 1902.

[53] E. N. Lorenz, "Empirical orthogonal functions and statistical weather prediction," Statistical Forecasting Project 1, MIT, Cambridge, Mass, USA, 1956.

[54] R. E. Davis, "Predictability of sea surface temperature and sea level pressure anomalies over the North Pacific Ocean," Journal of Physical Oceanography, vol. 6, no. 3, pp. 249-266, 1976.

[55] T. P. Barnett and K. Hasselmann, “Techniques of linear prediction, with application to oceanic and atmospheric fields in the tropical Pacific," Reviews of Geophysics \& Space Physics, vol. 17, no. 5, pp. 949-968, 1979.

[56] B. Wang and H. Yue, "EOF model analysis of place names landscape in Guangdong province on GIS," Scientia Geographica Sinica, vol. 27, no. 2, pp. 282-288, 2007.

[57] Z. Zhao, J. Wang, J. Zhao, and Z. Su, "Using a Grey model optimized by Differential Evolution algorithm to forecast the per capita annual net income of rural households in China," Omega, vol. 40, no. 5, pp. 525-532, 2012. 


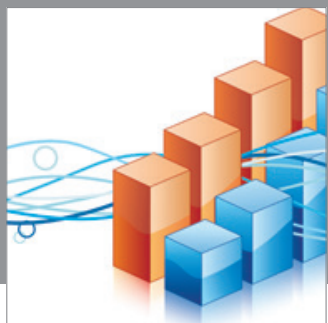

Advances in

Operations Research

mansans

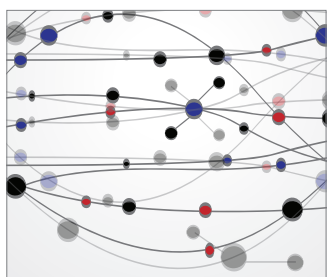

The Scientific World Journal
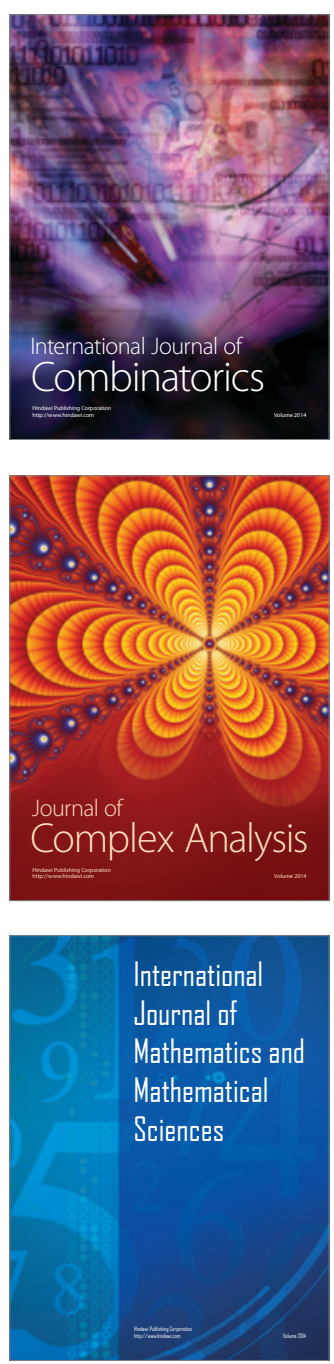
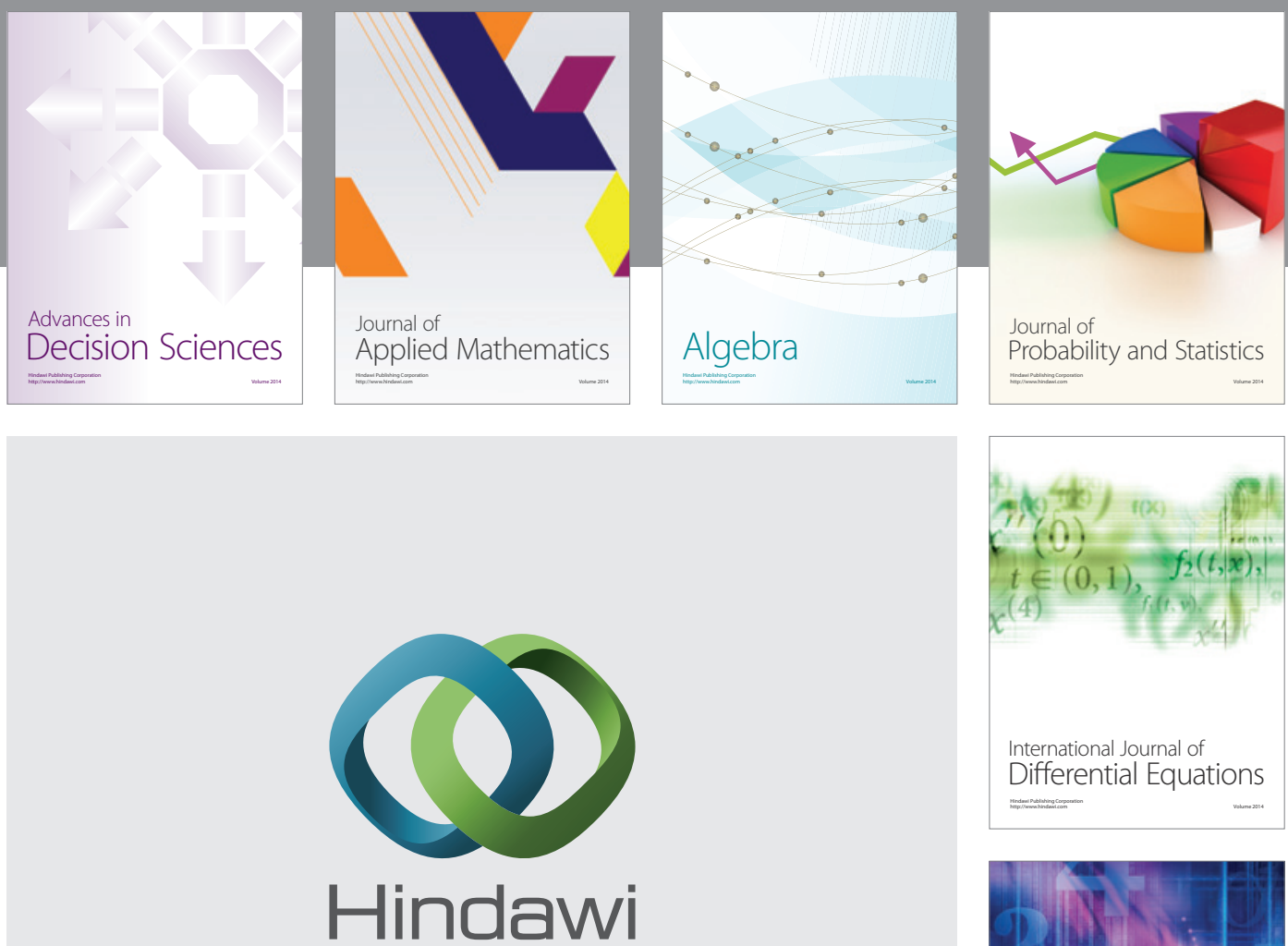

Submit your manuscripts at http://www.hindawi.com
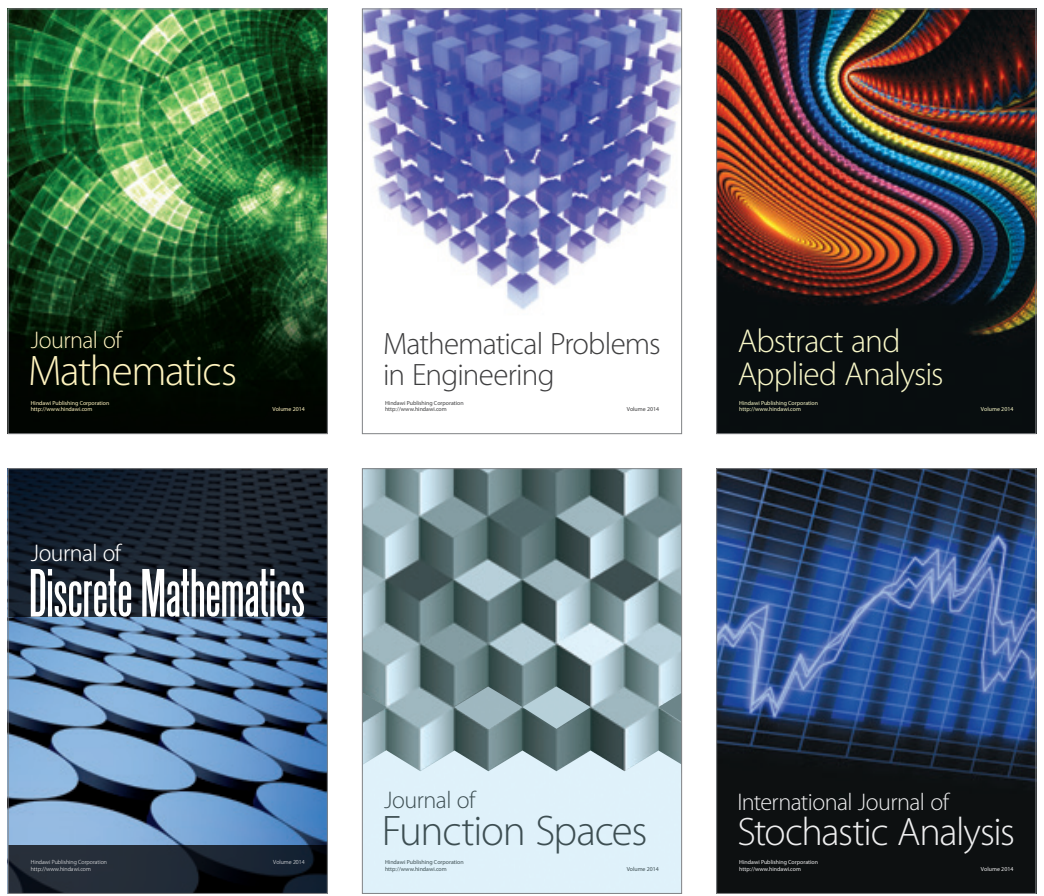

Journal of

Function Spaces

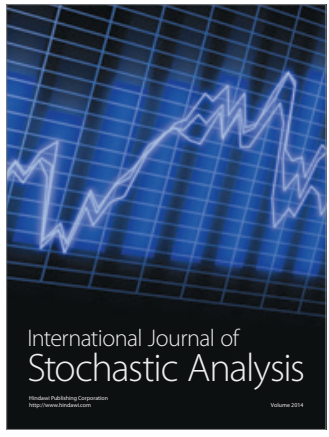

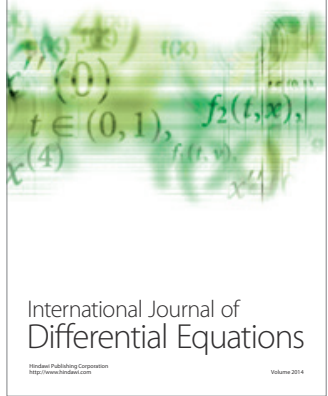
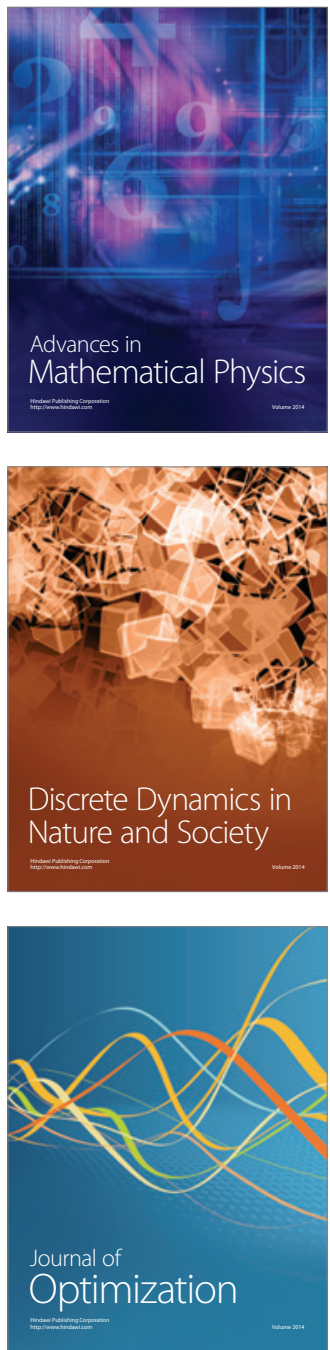